Journal of

Dentistry and Oral Health

\title{
Impact of Continuing Education on Clinicians' Self- Reported Knowledge of Tobacco Dependence and Tobacco Control Interventions
}

\section{Laura M. Romito, ${ }^{1, *}$, Lorinda Coan ${ }^{2}$}

${ }^{1}$ Associate Professor, Department of Biomedical and Applied Sciences, Indiana University School of Dentistry, 1121 W. Michigan St. Indianapolis, IN USA 44206

${ }^{2}$ Assistant Professor of Dental Hygiene, University of Southern Indiana, 8600 University Blvd. Room HP 2046 Evansville, IN 47712

${ }^{*}$ Corresponding author: Laura M. Romito, Associate Professor, Department of Biomedical and Applied Sciences Indiana University School of Dentistry, 1121 W. Michigan St. Indianapolis, IN USA 44206

Received Date: September 04, 2018; Accepted Date: October 18, 2018; Published Date: October 20, 2018

Citation: Laura M. Romito (2018) Impact of Continuing Education on Clinicians' Self- Reported Knowledge of Tobacco Dependence and Tobacco Control Interventions. J Dent Oral Health 5: 1-13.

\begin{abstract}
Purpose: To assess a tobacco cessation continuing education (CE) program for Indiana dental and medical providers. Methods: A 26-item immediate post-CE survey and a 19-item 3-month follow-up survey assessed changes in participants' self-reported knowledge of tobacco dependence and tobacco control interventions. De-identified data were analyzed using descriptive statistics, Spearman correlation coefficients, and Mantel- Haenszel chi-square tests.
\end{abstract}

Results: Participants totaled 252 across 6 programs statewide. Immediate post-CE course survey response was $98.4 \%$ $(\mathrm{N}=248)$ : dental assistants (2\%), dental hygienists (83\%), dentists (8.5\%), and other healthcare professionals (6.45\%). Participants reported less knowledge before than immediately after $\mathrm{CE}(\mathrm{p}<.0001)$ and 3 months after $(\mathrm{p}<.0001)$. Reported knowledge at 3 months was less than after $\mathrm{CE}(\mathrm{p}<.002)$. Participants reported on their intention to apply program communication strategies (99\%), implement brief tobacco interventions (85\%), and refer patients to local cessation resources (95\%), Indiana Quitline (96\%). Follow-up survey response rate was 54\% ( $=136)$. Participants reported active engagement in tobacco interventions (48\%, 78), applying CE communication strategies (85\%, 109), and implementing brief interventions (71\%, 90). Participants reported referring few patients to local or state quitline counselors. Conclusion:Tobacco dependence CE may enhance health care practitioners' knowledge and willingness to integrate tobacco interventions in their healthcare settings but it does not ensure a change in clinical tobacco control interventions.

C2018 The Authors. Published by the JScholar under the terms of the Creative Commons Attribution License http://creativecommons.org/licenses/by/3.0/, which loize.marechal@umontreal.ca

maximilien.laviolette-brassard@umontreal.ca 


\section{Introduction}

The principal cause of preventable disease and death in the United States is the use of tobacco.One in two smokers dies of tobacco-related diseases. [1] Tobacco use contributes to an extensive list of serious diseases, including cardiovascular and cerebrovascular diseases, cancers, emphysema, and bronchitis.Second-hand smoke is reported as a contributive factor in many pediatric illnesses such as asthma and others. The oral effects of tobacco use are also well documented and manifest as a wide variety of benign and serious conditions from dental stains to periodontal disease to oral and pharyngeal cancers; this renders tobacco use an important oral health concern. [1- 4] Moreover, tobacco use is responsible for nearly $\$ 170$ billion per year in direct medical costs in adults and in excess of $\$ 156$ billion per year in lost productivity due to exposure to environmental tobacco smoke and premature death $[1,5]$. It has been reported that fewer than $5 \%$ of tobacco users remain abstinent one year following a successful quit effort when attempted without assistance [6]. Healthcare personnel who give the advice to quit tobacco and offer pharmacotherapy can double or triple a client's success rate to stop and 'stay quit' compared to a tobacco user trying to quit by themselves [7]. Primary care clinicians such as medical and dental care providers have been encouraged by their respective professional organizations to implement proven tobacco cessation strategies within their practices. A national survey of health care providers during 2003-2004 found that 94.9\% of primary care physicians, $70.6 \%$ of dentists, and $77.5 \%$ of dental hygienists reported that they regularly advised their patients to stop smoking [8]. As 50\% of tobacco users have been reported to visit an oral health professional regularly [9], the oral health care provider is in a particularly unique position to provide tobacco cessation strategies to patients seen in their practices on a regular and on-going basis.

An analysis conducted by Danesh et. al. revealed that only $50 \%$ of current tobacco users reported having received advice to quit.Of those who did report being counseled, only 1 in 10 reported having heard the message from oral health care personnel [10]. Danesh et. al. [10] recognized a need for improvement from all health professions, but especially in dentistry, and suggested continuing education programs as one way to improve compliance with the U.S. Public Health Service (USPHS) Clinical Practice Guideline, Treating Tobacco Use and Dependence: 2008 Update. The USPHS Clinical Practice Guideline [7] provides clinicians with recommendations for helping tobacco users quit. The recommendations involve proven strategies such as behavioral counseling including the use of tobacco quitlines. Pharmacotherapy such as nicotine replacement therapy, varenicline, and bupropion are also recommended. Enhanced quit rate effectiveness has been shown with combinations of pharmacotherapy. The addition of counseling to the use of medications is even more effective in increasing quit rates [7].

It has been shown that training health care professionals to provide smoking cessation counseling favorably impacts professional performance [11]. Furthermore, patients expect oral health care providers to ask them about using tobacco [12]. Dolan and colleagues found that $14 \%$ of dentists and $23 \%$ of dental hygienists surveyed reported having completed formal tobacco cessation training [13]. More recent studies have found that $22-64 \%$ of oral healthcare practitioners have undergone training to help their patients quit tobacco $[14,15]$. Compared to those without training, oral health care providers with tobacco cessation training provide more tobacco use cessation services and advise more patients to stop using tobacco [13-16].

Oral healthcare providers typically have not been highly engaged in tobacco cessation counseling due to a number of perceived barriers [17]. The most commonly cited barriers include lack of time, lack of financial reimbursement, patient resistance/negativity, and a lack of confidence. Other reported barriers include inadequate knowledge of referral services available; focus on other healthcare needs; staff resistance to implement changes; inadequate training; and a lack of available educational materials $[15,18,19]$.

Gordon et. al. made the observation that no national standards exist for tobacco cessation curriculum in U.S. dental and allied dental schools. Furthermore, the Commission on Dental Accreditation does not consider tobacco cessation as a separate clinical competency [18]. Thus, for oral health professionals to systematically assist their patients to quit tobacco, changes must be made to the ways treatment of tobacco dependence is viewed and valued in practice. Until that time, the dental profession may continue to fall short of the USPHS Clinical Practice Guideline and the policies of oral health professions' organizations [18-21]. Nonetheless, oral healthcare environments continue to be an important, yet underused area for treating tobacco use and dependence, so much so that Healthy People 2020 has made a goal of improving the rates of tobacco screening and cessation counseling in dental care settings $[22,23]$.

Likewise, despite Guideline [7] recommendations, although most physicians routinely ask patients if they smoke and advise smokers to quit, less actually assist the patient 
throughout the quitting process $[24,25]$. The barriers often cited for not providing cessation interventions by medical colleagues are similar to those expressed by oral healthcare providers: a lack of both perceived efficacy and adequate training in tobacco control interventions [26-28].

Continued improvement in the efforts of health professionals to support the sustained delivery of tobacco cessation messages is warranted.Despite the availability of evidence-based tools like the Guideline [7], nationallyrecognized, clinician education programs, such as $\mathrm{Rx}$ For Change [29], and state toolkits [30, 31], as well as referral sources such as telephone quitlines [7,32] and cell phone applications [33] which have all been shown to be efficacious in helping patients quit tobacco, clinicians do not routinely provide tobacco interventions [34]. More needs to be done to educate clinicians on available resources, decrease the barriers, and enhance their self-efficacy in providing tobacco interventions.

This study aimed to 1) address several noted barriers to providing tobacco control interventions by offering a day-long, in-person, comprehensive continuing education (CE) course to healthcare professionals on tobacco use and dependence, 2) assess the effectiveness of the CE course at enhancing attendees' knowledge of tobacco's addictive nature and associated health effects; biomedical and behavioral tobacco interventions; local and statewide tobacco cessation referral resources; and the components and protocols for establishing a team-based approach for tobacco control interventions in practice, and 3) obtain information on the extent to which program participants' changed their tobacco intervention behaviors and access to community and statewide tobacco cessation resources following the program.

\section{Methods}

\section{Continuing Education (CE) Course Content}

The CE course entitled, "Tobacco Cessation in Clinical Practice-A Team Approach", was based on the USPHS Clinical Practice Guideline and developed by the authors who are educators with expertise in tobacco dependence education and treatment. The course sought to enhance Indiana healthcare practitioners' understanding of tobacco dependence and treatment and encourage them to provide tobacco control interventions. It was composed of a series of interactive lectures with skills-based practice in communication techniques, case studies in behavioral and biomedical interventions, and a question and answer session with the course instructors and local community partners of the Indiana State Department of Health (ISDH) Tobacco Prevention \& Cessation office. CE content included the following: the 5 A's (Ask, Advise, Assess, Assist, Arrange), and Ask, Advise \& Refer interventions that could be implemented in a time efficient manner; demonstration of motivational interviewing techniques for communicating with tobacco users to minimize resistance and motivate cessation efforts; where to obtain no/low cost patient education materials; introduction to and presentation by their local representative from the state Department of Health tobacco prevention and cessation office; reimbursement strategies for clinical tobacco interventions; and presentation of a team-based approach for implementing tobacco interventions in a healthcare setting. The course agenda from the program brochure is shown in Table 1.

The course was offered free of charge and provided 7 hours of continuing education credit towards Indiana licensure for physicians, physician assistants, nurses, respiratory therapists, dentists, dental hygienists, and dental assistants.

\section{CE Course Locations}

Investigators presented the course at the following six locations across the state of Indiana, USA: Goshen, Lafayette, Madison, Richmond, Tell City, and Vincennes.These locations were chosen because they were communities where the program had not been previously presented.Also taken into consideration were the tobacco use rates at these sites; most were higher than the state average as reported by the Indiana State Department of Health (Table 2).

Program informational brochures that included the agenda (Table 1) and registration information were mailed to all licensed oral health care providers and hospitals in a 50mile radius of the chosen sites.

\section{CE Course Assessment Methods}

A 26-item immediate post-CE survey and a 19-item, 3-month follow-up survey was developed by investigators to capture the participants' self-reported knowledge before, immediately after, and 3 months after the course, as well as planned changes in their tobacco control interventions as a result of the course, and tobacco intervention activities at 3 months following the course.

To establish content validity, each survey was piloted with 6 Indiana University School of Dentistry faculty; surveys were modified based upon pilot data. The 26-item immediate post-CE survey contained an item for each of the 9 tobacco dependence and treatment knowledge areas for which participants were asked to rate their knowledge on each of the survey item topics before versus after the course. The survey also contained 9 items regarding current biomedical 


\begin{tabular}{|c|c|c|}
\hline 8:00 a.m. $-8: 30$ am & Registration & \\
\hline $8: 30 \mathrm{am}-8: 35 \mathrm{am}$ & Introduction and Objectives & Lorinda L. Coan, LDH, MS \\
\hline $8: 35 a m-9: 35 a m$ & Nicotine Dependence 101 & Dr. Arden Christen \\
\hline $9: 35 a m-9: 45 a m$ & Break & \\
\hline $9: 45 \mathrm{am}-10: 15 \mathrm{am}$ & Oral health and Tobacco & Dr. Arden Christen, L. Coan, LDH, MS \\
\hline $10: 15 \mathrm{am}-11: 15 \mathrm{am}$ & Systemic Health and Tobacco & Dr. Stephen Jay \\
\hline $11: 15 \mathrm{am}-11: 30 \mathrm{am}$ & Molecular Biology of Tobacco & Dr. Jack Windsor \\
\hline $11: 30 \mathrm{am}-12: 15 \mathrm{pm}$ & Pharmacotherapy and Cessation Aids & Dr. Laura Romito \\
\hline $12: 15 \mathrm{pm}-1: 15 \mathrm{pm}$ & Lunch & \\
\hline $1: 15 \mathrm{pm}-2: 45 \mathrm{pm}$ & Behavioral Interventions & Lorinda L. Coan, LDH, MS \\
\hline $2: 45 \mathrm{pm}-3: 00 \mathrm{pm}$ & Break & \\
\hline $3: 00 \mathrm{pm}-3: 45 \mathrm{pm}$ & $\begin{array}{l}\text { The Office Model for Implementing } \\
\text { a Tobacco Cessation Program }\end{array}$ & Lorinda L. Coan, LDH, MS \\
\hline $3: 45 \mathrm{pm}-4: 15 \mathrm{pm}$ & Community Partners and Resources & $\begin{array}{l}\text { Department of Health County } \\
\text { Representatives }\end{array}$ \\
\hline $4: 15 \mathrm{pm}-4: 30 \mathrm{pm}$ & Course Wrap Up Lorinda & L. Coan, LDH, MS \\
\hline $4: 30 \mathrm{pm}$ & Adjournment & \\
\hline
\end{tabular}

Table 1. Agenda for the Continuing Education Course, "Tobacco Cessation in Clinical Practice-A Team Approach"

\begin{tabular}{|l|l|l|}
\hline CE Location & County & Smoking Rate (2011) \\
\hline Goshen & Elkhart & $21 \%$ \\
\hline Lafayette & Tippecanoe & $17 \%$ \\
\hline Madison & Jefferson & $28 \%$ \\
\hline Richmond & Wayne & $28 \%$ \\
\hline Tell City & Perry & $26 \%$ \\
\hline Vincennes & Knox & $31 \%$ \\
\hline Indiana (overall) & & $25.6 \%$ \\
\hline
\end{tabular}

Table 2: Tobacco Use Rates by Location of Continuing Education Course 
tobacco interventions and provision of patient resources, perceived barriers, and intention to implement tobacco control interventions with patients as a result of attending the program; one demographic item; and 7 items evaluating the program content and instructors. The format of survey items included multiple-choice with an option for write-in responses, 4-point scaled responses (great amount-moderate amount-slight amount-none) and 5-point-scaled responses (strongly agreeagree-undecided-disagree-strongly disagree).

The 3-month follow-up survey contained 9 knowledge items corresponding to those from the immediate post-CE survey, as well 9 items regarding participants' self-reported tobacco control interventions and perceived barriers since attending the CE course, and one demographic item. Approval for the project was obtained by the Indiana University Purdue University Indianapolis (IUPUI) Institutional Review Board (\#1208009443).

A total of 252 people attended the six CE programs. During each course, the study was explained to attendees and they were offered the opportunity to participate by agreeing to provide their contact information and complete the immediate and 3-month follow-up surveys. At the conclusion of each course, participants were asked to complete the immediate post-CE survey.

Three months after the date of the course, the followup survey was mailed or delivered electronically to those who had previously consented to participate and had completed the immediate post-CE survey. A contact information form, attached to the immediate survey was used to mail 3-month follow-up surveys and survey completion reminders to nonresponders. All attendees $(\mathrm{N}=252)$ were mailed a 19-item 3-month follow-up survey, cover letter, study information sheet, and self-addressed, stamped envelope. Following the initial mailing, two subsequent mailings were sent to nonresponders.

The survey was confidential; however, to track completion, both the contact information form and the surveys were coded with the same numerical identifier. Deidentified survey data were reviewed, coded and entered into an electronic database for analyses. Data analyses included descriptive statistic and comparisons of the same questions between times were made using Cochran-Mantel-Haenszel chisquare tests for repeated ordered categorical data. Associations of Immediate-Q13 with 3 month-Q14 and Immediate-Q14 with 3 month-Q15 were made using Mantel-Haenszel chisquare tests and Spearman correlation coefficients.

\section{Results}

The response rate for the immediate post-CE survey was $98.4 \%(\mathrm{~N}=248)$. Course participants who responded to the post-CE survey included: dental assistants (2\%); dental hygienists $(83 \%)$, dentists $(8.5 \%)$, and other healthcare professionals $(6.45 \%)$. The response rate for the 3 -month survey was $54 \%(\mathrm{~N}=136)$.

\section{Knowledge}

Table 3 summarizes the data for the knowledge items on both the immediate post-CE survey and the 3-month follow-up survey. Between time comparisons for all data showed less knowledge before than immediately after $(\mathrm{p}<.0001$ for all items) and 3 months after the CE course $(\mathrm{p}<.0001$, for all items); immediately after showed more knowledge than 3 months after the CE course for all survey questions (Q1:p=.0019;Q2-Q5:p<.0001; Q6:p=.0002; Q7:p=.0007; $\mathrm{Q} 9: \mathrm{p}=.0005)$ except question \#8 ( $\mathrm{p}=0.06)$.

\section{Tobacco Control Interventions}

Table 4 describes the participants' self-reported intention to implement tobacco control interventions immediately after the $\mathrm{CE}$ course, and three months later. Immediately after the CE course, $99 \%$ of participants ( $\mathrm{N}=247$ ) strongly agreed/agreed that the course improved their ability to use effective communication strategies with tobacco using clients; however, at 3 months post-CE, 85\% ( $\mathrm{N}=130)$ reported applying learned communication strategies. Similarly, immediately after the course $85 \%$ strongly agreed/agreed that they would implement the brief interventions from the course; at 3 months, however, $71 \%$ reported actually doing so. Immediately following the course, approximately $95 \%$ of responding participants indicated that they planned to refer interested patients to local tobacco cessation resources and the Indiana Tobacco Quitline. However, at 3 months 120 of $130(93 \%)$ participants reported referring 5 or fewer patients to local resources (Table 5) and 114 of 133 (88\%) referred 5 or fewer people to the Quitline (Table 6). There were no significant associations between participating clinicians' plans to refer to local tobacco cessation resources $(r=.16)$ and the Indiana Tobacco Quitline ( $\mathrm{r}=.02)$ and the number of patients referred at 3 months as reported by participants.

\section{Resources Provided}

Results for tobacco cessation resources provided to patients are shown in Table 7. 


\begin{tabular}{|c|c|c|c|c|c|c|c|}
\hline & Question & Total N & Great (1) & $\begin{array}{l}\text { Moderate } \\
\text { (2) }\end{array}$ & Slight (3) & None (4) & $\begin{array}{l}\text { Mean } \\
(\mathrm{SD})\end{array}$ \\
\hline \multirow[t]{9}{*}{ Before } & $\begin{array}{l}\text { Q1: Knowledge of oral effects of } \\
\text { tobacco }\end{array}$ & 248 & $46(19 \%)$ & $165(67 \%)$ & $37(15 \%)$ & $0(0 \%)$ & $2.0(0.6)$ \\
\hline & $\begin{array}{l}\text { Q2: Clear understanding nicotine } \\
\text { addiction }\end{array}$ & 248 & $23(9 \%)$ & $126(51 \%)$ & $98(40 \%)$ & $1(0 \%)$ & $2.3(0.6)$ \\
\hline & $\begin{array}{l}\text { Q3: Knowledge of pharm of NRT, } \\
\text { bupropion and varenicline }\end{array}$ & 247 & $7(3 \%)$ & $49(20 \%)$ & $\begin{array}{l}158 \\
(64 \%)\end{array}$ & $33(13 \%)$ & $2.9(0.7)$ \\
\hline & $\begin{array}{l}\text { Q4: Knowledge of NRT, } \\
\text { bupropion, varenicline dosing } \\
\text { requirements }\end{array}$ & 248 & $6(2 \%)$ & $27(11 \%)$ & $86(35 \%)$ & $129(52 \%)$ & $3.4(0.8)$ \\
\hline & $\begin{array}{l}\text { Q5: Knowledge of adverse effects } \\
\text { of NRT, Bupropion, varenicline }\end{array}$ & 246 & $7(3 \%)$ & $30(12 \%)$ & $\begin{array}{l}116 \\
(47 \%)\end{array}$ & $93(38 \%)$ & $3.2(0.8)$ \\
\hline & $\begin{array}{l}\text { Q6: Knowledge of communication } \\
\text { techniques for tobacco cessation }\end{array}$ & 247 & $10(4 \%)$ & $85(34 \%)$ & $\begin{array}{l}135 \\
(55 \%)\end{array}$ & $17(7 \%)$ & $2.6(0.7)$ \\
\hline & $\begin{array}{l}\text { Q7: Knowledge of selection of } \\
\text { community and state resources }\end{array}$ & 247 & $9(4 \%)$ & $35(14 \%)$ & $\begin{array}{l}143 \\
(58 \%)\end{array}$ & $60(24 \%)$ & $3.0(0.7)$ \\
\hline & $\begin{array}{l}\text { Q8: Clear understanding ISDH } \\
\text { local community resources } \\
\text { services }\end{array}$ & 246 & $5(2 \%)$ & $25(10 \%)$ & $\begin{array}{l}126 \\
(51 \%)\end{array}$ & $90(37 \%)$ & $3.2(0.7)$ \\
\hline & $\begin{array}{l}\text { Q9: Clear understanding of } \\
\text { Quitline service }\end{array}$ & 246 & $12(5 \%)$ & $26(11 \%)$ & $\begin{array}{l}101 \\
(41 \%)\end{array}$ & $107(43 \%)$ & $3.2(0.8)$ \\
\hline \multirow[t]{7}{*}{ Immediate } & $\begin{array}{l}\text { Q1: Knowledge of oral effects of } \\
\text { tobacco }\end{array}$ & 247 & $\begin{array}{l}211 \\
(85 \%)\end{array}$ & $35(14 \%)$ & $1(0 \%)$ & $0(0 \%)$ & $1.1(0.4)$ \\
\hline & $\begin{array}{l}\text { Q2: Clear understanding nicotine } \\
\text { addiction }\end{array}$ & 248 & $\begin{array}{l}203 \\
(82 \%)\end{array}$ & $44(18 \%)$ & $1(0 \%)$ & $0(0 \%)$ & $1.2(0.4)$ \\
\hline & $\begin{array}{l}\text { Q3: Knowledge of pharm of NRT, } \\
\text { bupropion and varenicline }\end{array}$ & 247 & $\begin{array}{l}149 \\
(60 \%)\end{array}$ & $91(37 \%)$ & $7(3 \%)$ & $0(0 \%)$ & $1.4(0.5)$ \\
\hline & $\begin{array}{l}\text { Q4: Knowledge of NRT, } \\
\text { bupropion, varenicline dosing } \\
\text { requirements }\end{array}$ & 247 & $\begin{array}{l}129 \\
(52 \%)\end{array}$ & $106(43 \%)$ & $12(5 \%)$ & $0(0 \%)$ & $1.5(0.6)$ \\
\hline & $\begin{array}{l}\text { Q5: Knowledge of adverse effects } \\
\text { of NRT, Bupropion, varenicline }\end{array}$ & 247 & $\begin{array}{l}135 \\
(55 \%)\end{array}$ & $103(42 \%)$ & $9(4 \%)$ & $0(0 \%)$ & $1.5(0.6)$ \\
\hline & $\begin{array}{l}\text { Q6: Knowledge of communication } \\
\text { techniques for tobacco cessation }\end{array}$ & 246 & $\begin{array}{l}183 \\
(74 \%)\end{array}$ & $60(24 \%)$ & $3(1 \%)$ & $0(0 \%)$ & $1.3(0.5)$ \\
\hline & $\begin{array}{l}\text { Q7: Knowledge of selection of } \\
\text { community and state resources }\end{array}$ & 247 & $\begin{array}{l}148 \\
(60 \%)\end{array}$ & $91(37 \%)$ & $8(3 \%)$ & $0(0 \%)$ & $1.4(0.6)$ \\
\hline
\end{tabular}




\begin{tabular}{|c|c|c|c|c|c|c|c|}
\hline & $\begin{array}{l}\text { Q8: Clear understanding ISDH } \\
\text { local community resources } \\
\text { services }\end{array}$ & 244 & $\begin{array}{l}120 \\
(49 \%)\end{array}$ & $105(43 \%)$ & $18(7 \%)$ & $1(0 \%)$ & $1.6(0.6)$ \\
\hline & $\begin{array}{l}\text { Q9: Clear understanding of } \\
\text { Quitline service }\end{array}$ & 245 & $\begin{array}{l}170 \\
(69 \%)\end{array}$ & $67(27 \%)$ & $8(3 \%)$ & $0(0 \%)$ & $1.3(0.5)$ \\
\hline \multirow[t]{9}{*}{3 month } & $\begin{array}{l}\text { Q1: Knowledge of oral effects of } \\
\text { tobacco }\end{array}$ & 136 & $90(66 \%)$ & $44(32 \%)$ & $2(1 \%)$ & $0(0 \%)$ & $1.4(0.5)$ \\
\hline & $\begin{array}{l}\text { Q2: Clear understanding nicotine } \\
\text { addiction }\end{array}$ & 135 & $75(56 \%)$ & $58(43 \%)$ & $2(1 \%)$ & $0(0 \%)$ & $1.5(0.5)$ \\
\hline & $\begin{array}{l}\text { Q3: Knowledge of pharm of NRT, } \\
\text { bupropion and varenicline }\end{array}$ & 136 & $21(15 \%)$ & $83(61 \%)$ & $32(24 \%)$ & $0(0 \%)$ & $2.1(0.6)$ \\
\hline & $\begin{array}{l}\text { Q4: Knowledge of NRT, } \\
\text { bupropion, varenicline dosing } \\
\text { requirements }\end{array}$ & 136 & $17(13 \%)$ & $60(44 \%)$ & $49(36 \%)$ & $10(7 \%)$ & $2.4(0.8)$ \\
\hline & $\begin{array}{l}\text { Q5: Knowledge of adverse effects } \\
\text { of NRT, Bupropion, varenicline }\end{array}$ & 136 & $18(13 \%)$ & $73(54 \%)$ & $40(29 \%)$ & $5(4 \%)$ & $2.2(0.7)$ \\
\hline & $\begin{array}{l}\text { Q6: Knowledge of communication } \\
\text { techniques for tobacco cessation }\end{array}$ & 136 & $71(52 \%)$ & $55(40 \%)$ & $10(7 \%)$ & $0(0 \%)$ & $1.6(0.6)$ \\
\hline & $\begin{array}{l}\text { Q7: Knowledge of selection of } \\
\text { community and state resources }\end{array}$ & 136 & $56(41 \%)$ & 60 (44\%) & $20(15 \%)$ & $0(0 \%)$ & $1.7(0.7)$ \\
\hline & $\begin{array}{l}\text { Q8: Clear understanding ISDH } \\
\text { local community resources } \\
\text { services }\end{array}$ & 135 & $56(41 \%)$ & $56(41 \%)$ & $20(15 \%)$ & $3(2 \%)$ & $1.8(0.8)$ \\
\hline & $\begin{array}{l}\text { Q9: Clear understanding of } \\
\text { Quitline service }\end{array}$ & 134 & $76(57 \%)$ & $42(31 \%)$ & $14(10 \%)$ & $2(1 \%)$ & $1.6(0.7)$ \\
\hline
\end{tabular}

Table 3: Clinicians' Self-Reported Tobacco Dependence and Treatment Knowledge Before, Immediately After, and 3 Months After the Tobacco CE Program

\begin{tabular}{|c|c|c|c|c|c|c|c|c|}
\hline Time & Question & $\begin{array}{l}\text { Total } \\
\mathrm{N}\end{array}$ & $\begin{array}{l}\text { Strongly } \\
\text { Agree (1) }\end{array}$ & $\begin{array}{l}\text { Agree } \\
(2)\end{array}$ & $\begin{array}{l}\text { Undecided } \\
\text { (3) }\end{array}$ & $\begin{array}{l}\text { Disagree } \\
(4)\end{array}$ & $\begin{array}{l}\text { Strongly } \\
\text { Disagree } \\
(5)\end{array}$ & $\begin{array}{l}\text { Mean } \\
(\mathrm{SD})\end{array}$ \\
\hline \multirow[t]{5}{*}{ Immediate } & $\begin{array}{l}\text { Q10: will improve ability to } \\
\text { play active role in team plan }\end{array}$ & 247 & $92(37 \%)$ & $\begin{array}{l}142 \\
(57 \%)\end{array}$ & $12(5 \%)$ & $0(0 \%)$ & $1(0 \%)$ & $1.7(0.6)$ \\
\hline & $\begin{array}{l}\text { Q11: will improve ability } \\
\text { to use communication } \\
\text { strategies }\end{array}$ & 247 & $115(47 \%)$ & $\begin{array}{l}128 \\
(52 \%)\end{array}$ & $3(1 \%)$ & $0(0 \%)$ & $1(0 \%)$ & $1.6(0.6)$ \\
\hline & $\begin{array}{l}\text { Q12: will implement the } \\
\text { brief tobacco intervention } \\
\text { strategies }\end{array}$ & 246 & $69(28 \%)$ & $\begin{array}{l}139 \\
(57 \%)\end{array}$ & $36(15 \%)$ & $2(1 \%)$ & $0(0 \%)$ & $1.9(0.7)$ \\
\hline & $\begin{array}{l}\text { Q13: will refer interested } \\
\text { patients to local resources }\end{array}$ & 246 & $102(41 \%)$ & $\begin{array}{l}132 \\
(54 \%)\end{array}$ & $10(4 \%)$ & $2(1 \%)$ & $0(0 \%)$ & $1.6(0.6)$ \\
\hline & $\begin{array}{l}\text { Q14: will refer interested } \\
\text { patients to the Indiana } \\
\text { Quitline }\end{array}$ & 244 & $129(53 \%)$ & $\begin{array}{l}104 \\
(43 \%)\end{array}$ & $9(4 \%)$ & $1(0 \%)$ & $1(0 \%)$ & $1.5(0.6)$ \\
\hline
\end{tabular}




\begin{tabular}{|l|l|l|l|l|l|l|l|l|}
\hline 3 month & $\begin{array}{l}\text { Q10: currently play active } \\
\text { role in team based tobacco } \\
\text { cessation }\end{array}$ & 130 & $10(8 \%)$ & $68(52 \%)$ & $22(17 \%)$ & $\begin{array}{l}22 \\
(17 \%)\end{array}$ & $8(6 \%)$ & $2.6(1.1)$ \\
\hline $\begin{array}{l}\text { Q11: applying the } \\
\text { communication strategies } \\
\text { learned in the course }\end{array}$ & 129 & $23(18 \%)$ & $86(67 \%)$ & $13(10 \%)$ & $4(3 \%)$ & $3(2 \%)$ & $2.1(0.8)$ \\
\hline & $\begin{array}{l}\text { Q12: implemented brief } \\
\text { tobacco intervention } \\
\text { strategies }\end{array}$ & 128 & $15(12 \%)$ & $75(59 \%)$ & $18(14 \%)$ & $\begin{array}{l}15 \\
(12 \%)\end{array}$ & $5(4 \%)$ & $2.4(1.0)$ \\
\hline
\end{tabular}

Table 4: Clinicians' Self-Reported Intention to Implement Tobacco Intervention Behaviors and Actual Implementation 3 Months Post-CE

\begin{tabular}{|l|l|l|l|l|l|l|l|}
\hline Time & Question & Total N & 0 & $1-5$ & $6-10$ & $11-15$ & $\begin{array}{l}16 \text { or } \\
\text { more }\end{array}$ \\
\hline 3 month & $\begin{array}{l}\text { Q14: \#pts referred to local } \\
\text { counselors since CE program }\end{array}$ & 130 & $62(48 \%)$ & $58(45 \%)$ & $8(6 \%)$ & $0(0 \%)$ & $2(2 \%)$ \\
\hline 3 month & $\begin{array}{l}\text { Q15: \# pts referred to Indiana } \\
\text { Quitline since CE program }\end{array}$ & 133 & $50(38 \%)$ & $64(48 \%)$ & $\begin{array}{l}12 \\
(9 \%)\end{array}$ & $3(2 \%)$ & $4(3 \%)$ \\
\hline
\end{tabular}

Table 5: Clinicians' Self-Reported Referrals to Local Cessation Resources and Indiana Tobacco Quitline 3 Months Post-CE

\begin{tabular}{|c|c|c|c|c|c|c|c|c|c|c|}
\hline \multicolumn{11}{|c|}{3 month } \\
\hline Referral & Immediate & $\begin{array}{l}\text { Total } \\
\mathrm{N}\end{array}$ & 0 & $1-5$ & $6-10$ & $11-15$ & $\begin{array}{l}16 \text { or } \\
\text { more }\end{array}$ & $\begin{array}{l}\text { Mean } \\
\text { (SD) }\end{array}$ & p-value & Correlation \\
\hline \multirow[t]{4}{*}{ Local } & $\begin{array}{l}\text { Strongly } \\
\text { Agree (1) }\end{array}$ & 58 & $\begin{array}{l}32 \\
(55 \%)\end{array}$ & $22(38 \%)$ & $4(7 \%)$ & $0(0 \%)$ & $0(0 \%)$ & $1.5(0.6)$ & 0.0700 & 0.16 \\
\hline & Agree (2) & 65 & $\begin{array}{l}28 \\
(43 \%)\end{array}$ & $31(48 \%)$ & $4(6 \%)$ & $0(0 \%)$ & $2(3 \%)$ & $1.7(0.8)$ & & \\
\hline & $\begin{array}{l}\text { Undecided } \\
\text { (3) }\end{array}$ & 3 & $0(0 \%)$ & $3(100 \%)$ & $0(0 \%)$ & $0(0 \%)$ & $0(0 \%)$ & $2.0(0.0)$ & & \\
\hline & Disagree (4) & 1 & $0(0 \%)$ & $1(100 \%)$ & $0(0 \%)$ & $0(0 \%)$ & $0(0 \%)$ & $2.0()$. & & \\
\hline \multirow[t]{4}{*}{ Quitline } & $\begin{array}{l}\text { Strongly } \\
\text { Agree (1) }\end{array}$ & 77 & $\begin{array}{l}30 \\
(39 \%)\end{array}$ & $36(47 \%)$ & $7(9 \%)$ & $1(1 \%)$ & $3(4 \%)$ & $1.8(0.9)$ & 0.7427 & 0.02 \\
\hline & Agree (2) & 49 & $\begin{array}{l}16 \\
(33 \%)\end{array}$ & $26(53 \%)$ & $4(8 \%)$ & $2(4 \%)$ & $1(2 \%)$ & $1.9(0.9)$ & & \\
\hline & $\begin{array}{l}\text { Undecided } \\
\text { (3) }\end{array}$ & 4 & $2(50 \%)$ & $1(25 \%)$ & $1(25 \%)$ & $0(0 \%)$ & $0(0 \%)$ & $1.8(1.0)$ & & \\
\hline & $\begin{array}{l}\text { Strongly } \\
\text { Disagree (5) }\end{array}$ & 1 & $\begin{array}{l}1 \\
(100 \%)\end{array}$ & $0(0 \%)$ & $0(0 \%)$ & $0(0 \%)$ & $0(0 \%)$ & $1.0()$. & & \\
\hline
\end{tabular}

Table 6: Numbers of Patients Referred to Local Cessation Resources and Indiana Tobacco Quitline Reported by Clinicians' 3 Months Post-CE 


\begin{tabular}{|c|c|c|c|c|c|}
\hline Time & Immedi & & Month & & $\mathrm{p}$-value \\
\hline Survey Question & Total N & Yes & Total N & Yes & \\
\hline Q15: Tobacco cessation resources are currently provided & 241 & $85(35 \%)$ & NA & NA & \\
\hline Q16: Resources-Literature display in reception area & 245 & $47(19 \%)$ & 133 & $32(24 \%)$ & \\
\hline Q16: Resources-Literature display in treatment area & 245 & $50(20 \%)$ & 133 & $44(33 \%)$ & $\mathrm{p}=0.0173$ \\
\hline Q16: Resources-Video in treatment area & 245 & $4(2 \%)$ & 133 & $4(3 \%)$ & \\
\hline Q16: Resources-Distributed directly to patient & 245 & $53(22 \%)$ & 132 & $66(50 \%)$ & $\mathrm{p}<.0001$ \\
\hline Q16: Resources-Practice website & 245 & $4(2 \%)$ & 133 & $4(3 \%)$ & \\
\hline Q16: Resources-Other & 245 & $11(4 \%)$ & 133 & $13(10 \%)$ & \\
\hline Q17: Barrier-Lack of time to distribute resources & 240 & $29(12 \%)$ & 133 & $27(20 \%)$ & \\
\hline Q17: Barrier-Lack of time to discuss resources & 240 & $48(20 \%)$ & 132 & $16(12 \%)$ & \\
\hline Q17: Barrier-Patient acceptance & 240 & $30(13 \%)$ & 132 & $39(30 \%)$ & $\mathrm{p}=0.0004$ \\
\hline Q17: Barrier-Lack of referral agencies in area & 240 & $19(8 \%)$ & 132 & $49(37 \%)$ & \\
\hline Q17: Barrier-Locating and obtaining resources & 240 & $49(20 \%)$ & 132 & $9(7 \%)$ & $\mathrm{p}=0.0003$ \\
\hline Q17: Barrier-Space for resource materials & 240 & $23(10 \%)$ & 132 & $10(8 \%)$ & \\
\hline Q17: Barrier-Cost of resource materials & 240 & $28(12 \%)$ & 132 & $17(13 \%)$ & \\
\hline Q17: Barrier-Other & 240 & $30(13 \%)$ & 132 & $11(8 \%)$ & \\
\hline Q18: Prescribe/Recommend NRT gum & 245 & $51(21 \%)$ & 132 & $17(13 \%)$ & $\mathrm{p}=0.0082$ \\
\hline Q18: Prescribe/Recommend NRT lozenge & 245 & $20(8 \%)$ & 132 & $30(23 \%)$ & $\mathrm{p}=0.0009$ \\
\hline Q18: Prescribe/Recommend NRT patch & 245 & $45(18 \%)$ & 132 & $55(42 \%)$ & $\mathrm{p}=0.0431$ \\
\hline Q18: Prescribe/Recommend NRT inhaler & 245 & $7(3 \%)$ & 132 & $30(23 \%)$ & \\
\hline Q18: Prescribe/Recommend NRT nasal spray & 245 & $5(2 \%)$ & 132 & $46(35 \%)$ & \\
\hline Q18: Prescribe/Recommend NRT bupropion & 245 & $13(5 \%)$ & 132 & $9(7 \%)$ & \\
\hline Q18: Prescribe/Recommend NRT varenicline & 245 & $30(12 \%)$ & 132 & $4(3 \%)$ & $\mathrm{p}=0.0330$ \\
\hline Q18: Prescribe/Recommend no pharmacotherapy & 245 & $135(55 \%)$ & 132 & $16(12 \%)$ & \\
\hline
\end{tabular}

Table 7: Clinician's Self-Reported Tobacco Cessation Resources and Perceived Barriers Before and 3 Months Post-CE 
Immediate to 3 months comparisons showed increases for providing any resources $(\mathrm{p}<.0001)$, literature display in the treatment area $(\mathrm{p}=0.0173)$, distribution directly to patient $(\mathrm{p}<.0001)$, patient acceptance as a barrier $(\mathrm{p}=0.0004)$, and prescription or recommendation of NRT gum $(\mathrm{p}=0.0082)$, lozenge $(\mathrm{p}=0.0009)$, and patch $(\mathrm{p}=0.0431)$.

Comparisons between times using all data showed decreases from immediate to 3 months for locating and obtaining resources as a barrier $(\mathrm{p}=0.0003)$ and prescription/ recommendation of NRT, varenicline $(\mathrm{p}=0.0330)$.

\section{Program Evaluation}

The CE course was well-received. Of the responding participants $(\mathrm{N}=238)$, approximately $75 \%$ "strongly agreed" that the instructors demonstrated expertise and presented high-quality scientific content based on the best available evidence, presented appropriately for the target audience and related to attendees current scope of practice, and presented information in an organized and clear manner.

Of responding participants $(\mathrm{N}=235), 52 \%$ strongly agreed, and $47 \%$ agreed that the scope of the program was appropriate for their needs. Concerning whether the course held their interest, of 241 participants, 25\% strongly agreed and $64 \%$ agreed that it did and 54\% strongly agreed while $44 \%$ agreed that it was a worthwhile investment of their time. Of the participants answering the question concerning course resource materials $(\mathrm{N}=240), 69 \%$ strongly agreed and 39\% agreed that the program provided helpful and supporting materials and tools for enhancing their ability to provide tobacco interventions.

\section{Discussion}

Previous research has indicated that compared to medical providers, oral healthcare professionals are less likely to assist patients in tobacco cessation efforts but that training may improve the likelihood that they would do so. [13-16, 25] Thus, the overall goal of the comprehensive CE course, "Tobacco Cessation in Clinical Practice - a Team Approach" was to enhance clinicians' engagement, particularly oral health care providers, in tobacco control interventions in their practices. The course aimed to accomplish this goal by enhancing participants' knowledge of 9 key topic areas in tobacco dependence and treatment, demonstrating and practicing communication techniques through interactive skills training, providing potential solutions to common barriers, and describing the team-based approach to tobacco control interventions and its associated benefits for the clinician and the patient.
Participants rated the course highly; they perceived that it provided valuable information and enhanced their ability to provide tobacco control interventions in their practices. Furthermore, the results of the self-reported knowledge assessment indicated that attendees believed that the course did improve their understanding of tobacco use and dependence as well as biomedical and behavioral tobacco interventions. However, participants' self-reported knowledge at three months following the program decreased, although not to pre-program levels. This is consistent with the literature indicating that some degree of knowledge decay is to be expected over time following instruction. [35, 36] Nonetheless, the program did improve clinicians' self-reported knowledge and positive attitudes concerning tobacco control interventions in practice settings. Sheffer [37] found similar changes in perceived knowledge and attitudes following only a 1-hour tobacco training session for healthcare providers with the dental clinicians showing the greatest improvement. Likewise, Walsh and colleagues [16] noted that compared to those without training, dental providers who had engaged in a day-long tobacco training workshop were more likely to apply Guideline [7] recommendations and have favorable perceptions of tobacco cessation counseling.

Regarding changes in clinicians' tobacco control interventions, despite the perception that the course enhanced their ability to refer receptive tobacco users to local and statewide resources, at three months following the program, clinicians reported referring very few patients for tobacco cessation counseling. This may have been due to a number of factors, for example, clinicians may have encountered few tobacco users who interested in accepting the referral. Nonetheless, Ebbert et al [38] showed that if oral healthcare providers are aware of and comfortable with tobacco quitline services, such referrals by dental practices is an effective strategy to address tobacco use and dependence.

Study outcomes suggest that participants increased the display and dissemination of tobacco education and cessation materials, as well as the recommendation of nicotine replacement therapy (NRT) to patients, particularly the over-the-counter medications. This would suggest that the course influenced some of the practitioners' tobacco control intervention behaviors by improving their awareness, procurement, and utilization of patient education resource materials as well as enhancing their confidence in discussing tobacco cessation pharmacotherapy.

Research indicates that knowledge transfer is complex and may be best viewed as an ongoing process rather 
than a single event [39]. Thus, while motivation and training increase the likelihood of initial adoption of an innovation and its early use, other factors, such as ongoing access to information and support for the innovation, are beneficial to promote continued adoption [40]. In addition, while training workshops have shown some impact on practice [41], clinical practice behavior is minimally influenced through training alone; clinical training combined with ongoing coaching or "booster sessions" can enhance and sustain practice change [42]. Clinical education that focuses on enhancing providers' intrinsic motivation for change and identifies and overcomes perceived barriers, provides skill training with directed practice, and engages practitioners in ongoing support for change (e.g., networking, ongoing coaching, communities of practice) can more effectively promote adherence to evidence-based models and tools $[43,44]$. Therefore, the course may have a greater impact on clinician behaviors if follow-up sessions or ongoing support were offered. However, the course sought to address this issue to some degree by introducing participants to local ISDH cessation counselors and resources such as the Indiana Quitline Preferred Provider program which offers support and partners with the clinicians in their community to help tobacco users' quit. It was anticipated that gaining awareness of these resources and meeting their local representatives would encourage providers to network and become comfortable with using them as a referral source for their patients.

The self-reported nature of data is a limitation of the current study.Research has indicated that clinicians are inclined to overstate their engagement in tobacco interventions [45], therefore, our immediate and 3-month post-program results should be interpreted cautiously. Secondly, although the current study found increases in clinicians' self-reported tobaccorelated knowledge and tobacco control interventions, there is no evidence that clinician tobacco interventions changed in the long term as a result of the participating in the course. Additionally, it cannot be assumed that any such changes had an impact on their patient quit rates. The practitioners who chose to attend the course may have had a particular interest in tobacco dependence and treatment interventions and so were more motivated to learn and adopt such interventions in their practices.It is also possible that participants who responded to the 3-month follow-up survey may have been among the most interested and most likely to integrate knowledge, skills, and abilities obtained in the program into clinical practice. Despite these limitations, however, study outcomes indicated that overall, the course was beneficial in that it improved clinicians' perception of their knowledge about tobacco use and dependence, and may have facilitated an increase in their tobacco control interventions.

\section{Conclusion}

Continuing education on tobacco use, dependence and treatment is beneficial, at least in the short-term, in enhancing health care practitioners knowledge and willingness to integrate tobacco control interventions in their healthcare settings. However, this does not ensure that they will substantially change their practice behaviors by utilizing the learned concepts and tobacco interventions with patients.

\section{Acknowledgements}

The authors wish to thank the CE course instructors Dr. Arden Christen, Dr. Stephen Jay, and Dr. Jack Windsor, as well as the Indiana State Department of Health and the Indiana Dental Hygienists' Association for their contributions to this program.

This project was funded by a grant of the Indiana State Department of Health Tobacco Prevention and Cessation. 


\section{References}

1. (2014) U.S. Department of Health and Human Services. The Health Consequences of Smoking - 50 Years of Progress: A Report of the Surgeon General. Atlanta: U.S. Department of Health and Human Services, Centers for Disease Control and Prevention, National Center for Chronic Disease Prevention and Health Promotion, Office on Smoking and Health.

2. Albert D, Ward A (2012) Tobacco Cessation in the Dental Office. Dent Clin N Am 56:747-770.

3. (2007)World Health Organization. IARC Monographs on the Evaluation of Carcinogenic Risks to Humans. Volume 89: Smokeless Tobacco and Some Tobacco-Specific N-Nitro Lyon (France): World Health Organization, International Agency for Research on Cancer, [accessed 2015 July 1].

4. Piano MR, Benowitz NL, Fitzgerald GA, Corbridge S, Heath J,et al. (2010) Impact of Smokeless Tobacco Products on Cardiovascular Disease: Implications for Policy, Prevention, and Treatment: A Policy Statement from the American Heart Association. Circulation 122:1520-1544.

5. Xu X, Bishop EE, Kennedy SM, Simpson SA, Pechacek TF (2014) Annual Healthcare Spending Attributable to Cigarette Smoking: An Update Am J Prev Med 48:326-333.

6. Hammond D, McDonald PW, Fong GT, et al. (2004) Do smokers know how to quit? Knowledge and perceived effectiveness of cessation assistance as predictors of cessation behavior. Addiction 99:1042-1048.

7. Fiore MC, Jaen CR, Baker TB, et al. (2008) Treating tobacco use and dependence: clinical practice guideline. Rockville (MD): U.S. Department of Health and Human Services, Public Health Service.

8. Tong EK, Strouse R, Hall J, Kovac M, Schroeder S (2010) National survey of U.S. health professionals' smoking prevalence, cessation practices, and beliefs. Nicotine Tob Res 12:724-733.

9. Mucci LA, Brooks DR (2001) Lower use of dental services among long term cigarette smokers. J Epidemiol Community Health 55:389-393.

10. Danesh D, Paskett ED, Ferketich AK (2014) Disparities in Receipt of Advice to Quit Smoking from Health Care Providers: 2010 National Health Interview Survey. Prev Chronic Dis 11:140053.

11. Lancaster T., Silagy C, Fowler G (2000) Training health professionals in smoking cessation. Coch Data Sys Reviews 3:CD000214.

12. Christen AG (2001) Tobacco cessation, the dental profession, and the role of dental education. J Dent Educ 65:368-374.

13. Dolan TA, McGorray SP, Grinstead-Skigen CL, Mecklenburg R (1997) Tobacco control activities in U.S. dental practices. J Am Dent Assoc 128:1669-1679.

14. Jannat-Khah DP, McNeely J, Pereyra MR, Parish C, Pollack HA, et al. (2014) Dentists' self-perceived role in offering tobacco cessation services: results from a nationally representative survey, United States, 2010-2011.Prev Chronic Dis 6: 11:E196.
15. Patel AM, Blanchard SB, Christen AG, Bandy RW, Romito LM (2011) A survey of United States periodontists' knowledge, attitudes, and behaviors related to tobacco-cessation interventions. J Periodontol 82:367-376.

16. Walsh M, Belek M, Prakash P, Grimes B, Heckman B, et al. (2012) The effect of training on the use of tobacco-use cessation guidelines in dental settings. J Am Dent Assoc143:602-613.

17. Albert DA, Severson H, Gordon J, Ward A, Andrews J,et al. (2005) Tobacco attitudes, practices, and behaviors: a survey of dentists participating in managed care. Nicotine Tob Res 7 Suppl 1:S9-18.

18. Gordon JS, Severson HH (2001) Tobacco cessation through dental office settings. J Dent Educ 65:354-336.

19. Monson AL (2004) Barriers to Tobacco Cessation Counseling and Effectiveness of Training. Journal of Dental Hygiene, Vol. 78, No. 3, Summer.

20. Gordon JS, Andrews JA, Crews KM, Payne TJ, Severson $\mathrm{HH}$, e3t al. (2013) Do faxed quitline referrals add value to dental officebased tobacco-use cessation interventions? J Am Dent Assoc.

21. Coan L, Windsor LJ, Romito LM (2015) Increasing Tobacco Intervention Strategies by Oral Health Practitioners in Indiana. J Dent Hyg 89:190-201.

22. Gordon JS, Lichtenstein E, Severson HH, Andrews JA (2006) Tobacco cessation in dental settings: research findings and future directions. Drug Alcohol Rev 25:27-37.

23. US Department of Health and Human Services. Healthy people 2020 topics and objectives: tobacco use.

24. Goldstein M, DePue J, Monroe A, Lessne C, Rakowski W, et al. (1998) A population-based survey of physician smoking cessation counseling practices. Prev Med 27:720-729.

25. Applegate BW, Sheffer CE, Crews KM, Payne TJ, Smith $\mathrm{PO}(2008)$ A survey of tobacco-related knowledge, attitudes and behaviours of primary care providers in Mississippi. J Eval Clin Pract. 14: 537-544.

26. Vogt F, Hall S, Marteau TM (2005) General practitioners' and family physicians' negative beliefs and attitudes towards discussing smoking cessation with patients: a systematic review. Addiction 100:1423-1431.

27. Sarna LP, Brown JK, Lillington L, Rose M, Wewers ME, et al. (2000) Tobacco interventions by oncology nurses in clinical practice: report from a national survey. Cancer 89:881-889.

28. Twardella D, Brenner H (2005) Lack of training as a central barrier to the promotion of smoking cessation: a survey among general practitioners in Germany. Eur J Public Health 15:140-1455.

29. Prochaska JJ, Benowitz NL, Glantz SA, Hudmon KS, Grossman W (2003) Cardiology Rx for Change: improving clinical attention to tobacco use and secondhand smoke exposure in cardiology. Clin Cardiol 34: 738-743.

30. Indiana Dental Association. Tobacco Cessation Toolkit for Indiana Dental Practices. 
31. Texas Department of State Health Services. Clinical Toolkit for Treating Tobacco Dependence.

32. Stead LF, Hartmann-Boyce J, Perera R, Lancaster T (2013) Telephone counselling for smoking cessation. Cochrane Database Syst Rev 12: CD002850.

33. Whittaker R1, McRobbie H, Bullen C, Rodgers A, Gu Y (2016) Mobile phone-based interventions for smoking cessation. Cochrane Database Syst Rev 10;4:CD006611.

34. Tong EK, Strouse R, Hall J, et al. (2010) National survey of U.S. health professionals' smoking prevalence, cessation practices, and beliefs. Nicotine Tob Res 12: 724-733.

35. Crofts JF, Fox R, Draycott TJ, Winter C, Hunt LP, Akande VA (2013) Retention of factual knowledge after practical training for intrapartum emergencies. Int J GynaecolObstet123: 81-5.

36. Settles J, Jeffries PR, Smith TM, Meyers JS (2011) Advanced cardiac life support instruction: do we know tomorrow what we know today? J Contin Educ Nurs.42: 271-279.

37. Sheffer CE, Barone CP, Anders ME (2009) Training health care providers in the treatment of tobacco use and dependence: preand post-training results. J Eval Clin Pract.15:607-613.

38. Ebbert JO, Carr AB, Patten CA, Morris RA, Schroeder DR (2007)Tobacco use quitline enrollment through dental practices: a pilot study.J Am Dent Assoc138:595-601.

39. Greenhalgh T, Robert G, Macfarlane F, Bate P, Kyriakidou O (2004) Diffusion of innovations in service organizations: systematic review and recommendations. Milbank Q 82:581-629.

40. Herie M, Connolly H, Voci S, et al. (2012) Changing practitioner behavior and building capacity in tobacco cessation treatment: The TEACH project Patient Education and Counseling 86 49-56.

41. Lancaster T, Silagy C, Fowler G (2000) Training health professionals in smoking cessation. Cochrane Database Syst Rev 3.

42. Miller W, Sorensen J, Selzer J, Brigham G (2006) Disseminating evidence-based practices in substance abuse treatment: a review with suggestions. J Subst Abuse Treat 31:25-39.

43. Straus SE, Tetroe J, Graham I (2009) Defining knowledge translation. CMAJ 181:165-168.

44. Miller W, Mount K (2001) A small study of training in motivational interviewing: does one workshop change clinician and client behavior? Behav Cognitive Psychother 29:457-471.

45. Conroy M, Majchrzak N, Silverman C, Chang Y, Regan S (2005) Measuring provider adherence to tobacco treatment guidelines: a comparison of electronic medicalrecord review, patient survey, and provider survey. Nicotine and Tob Res 7 S35-S43.

\section{Submit your manuscript to a JScholar journal} and benefit from:

ฯ Convenient online submission

q Rigorous peer review

- Immediate publication on acceptance

- Open access: articles freely available online

ब High visibility within the field

a Better discount for your subsequent articles Submit your manuscript at http://www.jscholaronline.org/submit-manuscript.php 